\title{
Research and Development of the eXtendable Solar Array System
}

\author{
Nathan McKay ${ }^{1}$, Andrew Chou ${ }^{1}$, Daniel Becker ${ }^{1}$, Clark Hoffman ${ }^{1}$, Jeffery Walters ${ }^{1}$ \\ University of Michigan, Ann Arbor, MI, 48105
}

The eXtendable Solar Array System (XSAS) is a CubeSat power generation and distribution system under development by a team of students at the University of Michigan. Low power generation is a major factor limiting current CubeSat capabilities. XSAS is being designed as a $1 \mathrm{U}(10 \mathrm{~cm} \times 10 \mathrm{~cm} \times 10 \mathrm{~cm})$ CubeSat attachment capable of high power generation by utilizing an extending array of solar panels that provide a large surface compared to the common $3 \mathrm{U}(10 \mathrm{~cm} \times 10 \mathrm{~cm} \times 30 \mathrm{~cm})$ CubeSat. The geometry of extendable structure can provide additional benefits to a mission such as passive gravity gradient stabilization and the potential to integrate with a high gain antenna for enhanced communication capabilities. Preliminary calculations on the baseline design show that XSAS provides more than 20 watt-hours average power at $100^{\circ}$ inclination orbits above $600 \mathrm{~km}$, nearly triple the average 7 watt-hours power generation capability of a $3 \mathrm{U}$ CubeSat. A scaled down version of the extending array was tested in microgravity and validated as a reliable design. Currently, the XSAS project is in a research and development stage. All aspects of the XSAS technology are being investigated or redesigned based on previous testing results, lessons learned, and trade studies. Modeling is being done to determine power generation capabilities with respect to a range of orbital altitude and inclinations, structural characteristics, and thermal management methods. The extending structure and electrical power system are being designed and prototyped. The group will continue to raise the Technology Readiness Level and one day utilize XSAS to benefit a CubeSat mission.

$\begin{array}{ll}\text { XSAS } & \text { Nomenclature } \\ 1 \mathrm{U} & =\text { Ontendable Solar Array System } \\ 3 \mathrm{U} & =\text { Three CubeSat units or }(10 \mathrm{~cm} \times 10 \mathrm{~cm} \times 30 \mathrm{~cm}), \text { maximum allowed CubeSat size } \\ \mathrm{EPS} & =\text { Electrical Power System } \\ \mathrm{MNP} & =\text { Michigan NanoSat Pipeline } \\ \mathrm{PCB} & =\text { Printed Circuit Board } \\ \mathrm{MPPT} & =\text { Maximum Power Point Tracker } \\ \mathrm{I}^{2} \mathrm{C} & =\text { Inter-Integrated Circuit }\end{array}$

\section{Introduction}

T imited power is the main factor restricting the capabilities of CubeSat ${ }^{1}$ missions. Without drastically improving Lthe efficiencies of the body-mounted solar cells, an upper limit of power generation is reached due to the limited surface area of the standardized CubeSat. With growing popularity of CubeSats in academic and professional communities, methods to increased power generation have become a major concern. Deployable structures offer a solution to improve power generation. Making use of small mechanisms, deployable panels increase the available surface area for solar cells, easily doubling power generation capabilities.

The eXtendable Solar Array System (XSAS) offers a unique solution to increase available power generation surface area, and provide passive gravity gradient stabilization, without restricting the overall design of the CubeSat mission it is supporting. Unlike previously developed deployable solar arrays, which span the entire side of the CubeSat, XSAS extends linearly from a $1 \mathrm{U}(10 \mathrm{~cm} \times 10 \mathrm{~cm} \times 10 \mathrm{~cm})$ stowed configuration to an overall length of 1.1 meters. This grants the mission designers flexibility in the structural design of their CubeSat. Computer

\footnotetext{
${ }^{1}$ Undergraduate Student, University of Michigan Aerospace Engineering, 1320 Bean Ave, Ann Arbor, MI, 48105, Student Membership.
} 
simulations show that XSAS provides more than 20 watt-hours average power at $100^{\circ}$ inclination orbits above 600 $\mathrm{km}$, nearly triple the average 7 watt-hours power generation capability of a $3 \mathrm{U}(10 \mathrm{~cm} \times 10 \mathrm{~cm} \times 30 \mathrm{~cm})$ CubeSat without deployable surfaces. In addition to increased power generation, the solar array structure provides passive gravity gradient stabilization that forces the CubeSat to become nadir pointing. The structure can also be integrated with a high gain antenna for enhanced communication capabilities. Due to the use of mechanisms and the inherit complexity of a large solar array, research and testing are being done to buy down risk of the XSAS technology.

The XSAS team is currently entering the build phase of the extending structure's third iteration, developing the first iteration of the Electrical Power System (EPS), and investigating the performance of the system through computer simulation. The XSAS structure has previously been through two major design-built-test cycles. Past designs of the XSAS solar array structure are shown in Fig. 1. Computer modeling is being used to determine performance characteristics such as power generation and gravity gradient stabilization, thermal management, structural dynamics, and lifetime degradation due to the space environment. Furthermore, the EPS is now being designed, using heritage from current University of Michigan CubeSat missions. This paper presents the most recent design of the extending solar array mechanism and an initial design of the EPS system.

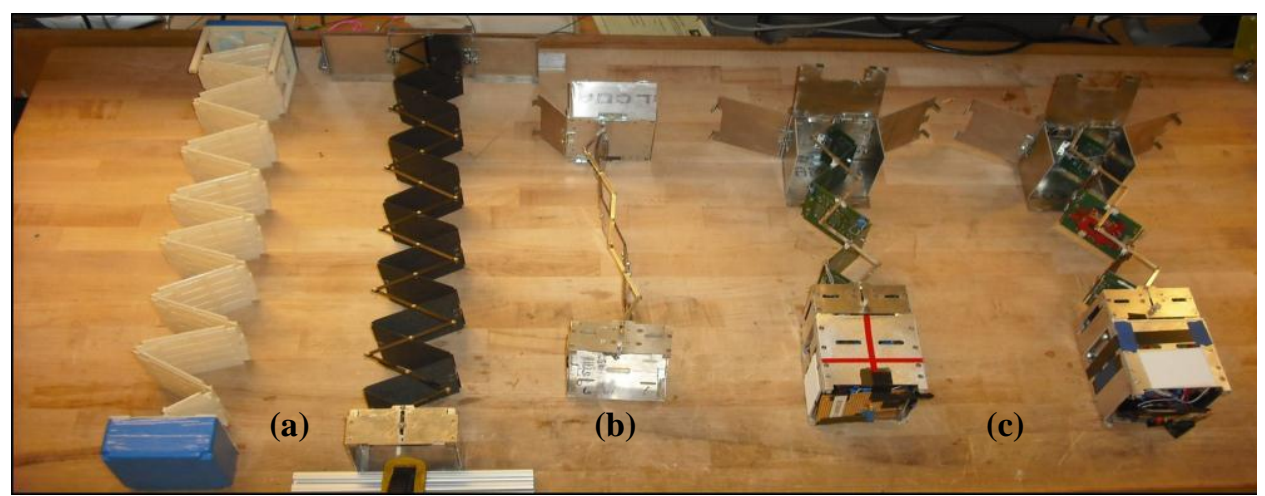

Figure 1. Past two design iterations of XSAS structure. Five structures have been built and tested: a) the initial 3D printed prototype and final full scale proof of concept assembly, $b$ ) the prototype of the scaled down structure, $c$ ) and the two scaled down flight units that were tested in microgravity.

\section{Background}

XSAS was originally conceptualized in an intensive year-long graduate student effort at the University of Michigan and designed to be assimilated into the Michigan NanoSat Pipeline ${ }^{2}$ (MNP), a program established to sustain the growth of small satellite projects within the university and to launch and develop new and innovative space technologies. Full scale prototypes of XSAS were manufactured and tested as proof of concept demonstrations, as shown in Fig. 1a. XSAS will be easily adaptable to any MNP mission and act as a technology enabler for the CubeSat, allowing for the use of advanced payloads and more flexible mission design.

Since the establishment of the CubeSat standard in 1999, there have been several proposals for deployable systems on CubeSats, but few have been successful. There are deployment risks associated with space-based mechanisms. Of the few CubeSats that have successfully incorporated the use of deployable systems, one of the most notable is QuakeSat ${ }^{3}$, developed by Stanford University and launched in 2003. QuakeSat had deployable tape spring antennas, a telescoping boom for magnetometer payload-to-CubeSat separation, and two deployable solar array "wings". These solar arrays were housed outside the CubeSat and spanned its entire length. They were attached to the structure at one end with single barrel spring hinges. Delfi- $\mathrm{C}^{4}$, developed by Delft University in the Netherlands and launched in 2008, was also successful in using four similar deployable solar array wing design as well as deployable antennas.

There are several current technologies in development that focus on increased power generation through deployable arrays. The Solar Array Drive ${ }^{5}$ from HoneyBee Robotics uses two motorized deployable arrays that are capable of active sun tracking. Active sun tracking is necessary in order to achieve high levels of power generation without large solar panel area, but requires additional power to run the tracking systems and sensors. It also has 
increased mechanical risk from continually moving parts in the drive. Pumpkin Inc., the makers of the CubeSat Kit, now have a seven panel solar array ${ }^{6}$. The seven panels of the array, each being approximately $10 \mathrm{~cm} \times 30 \mathrm{~cm}$ in size, are configured to fold around a $3 \mathrm{U}$ CubeSat when stowed and deploy to form one large surface. While capable of high power generation, these designs do not include means for orbital stabilization as XSAS does.

There are also technologies in development that, similar to XSAS, combine power generation with passive gravity gradient stabilization. Gravity gradient is a popular choice of passive stabilization as it can be easily integrated and miniaturized for CubeSats; it is only dependent on CubeSat mass and geometry. This type of stabilization forces the CubeSat to become nadir pointing. Foster-Miller and Qinetiq are developing a deployable Gravity Gradient Solar Array Boom or G-Sab ${ }^{7}$. It uses a flexible composite material that connects two $1.5 \mathrm{U}$ CubeSats and folds up around the structures when stowed. Upon deployment, the potential energy stored in the material allows it to unfold into two meter long boom with sufficient area for placement of solar cells. Tethers Unlimited $^{8}$ makes long deployable tethers with attached sensors on the ends. The long tether with deployed mass is sufficient to produce gravity gradient stabilization. In addition, the long conductive tether being dragged through the Earth's magnetosphere induces small currents that can be used for trickle charging and occasional bursts of power.

During 2010, XSAS underwent small scale deployment testing in a $1 \mathrm{~g}$ lab environment and during a $0 \mathrm{~g}$ microgravity flight through NASA's Microgravity University Program. Several XSAS prototypes were manufactured for use in microgravity flight testing and ground testing (Fig. 1 b \& c). Strain gauges, accelerometers, and IMUs were mounted on the XSAS structure. For ground testing, a rig was fabricated to study deployment of XSAS as it extends horizontally on a smooth surface. Tests were performed on a sheet of ice, providing three degrees of freedom. Video data was analyzed to determine deployment times. Unlike ground testing, a microgravity environment provided the unique opportunity to test the deployment of XSAS in six degrees of freedom.

The microgravity flights provided 60 microgravity testing periods, with each period lasting approximately 18-20 seconds. An aircraft mounted test chassis was built to rotate XSAS at 0,30 , and 60 degrees per second, simulating the residual rotation of a deployed CubeSat in orbit, and release it into microgravity. Of the 60 periods, data was successfully captured during 41 attempts. Data was captured by the sensors attached to the XSAS structure as well as from video cameras facing the front and side of the CubeSat. Unfortunately, due to a malfunction during flight, the test chassis was unusable and XSAS was instead rotated and released by hand. Test data was analyzed post flight to determine the forces and moments acting on the XSAS structure and the frequencies it undergoes during deployment. Data from microgravity testing was compared with ground testing and ADAMS simulation results ${ }^{8}$.

\section{Current Work}

\section{A. Mechanical Structure}

The XSAS team has finalized the design for the third iteration of the deployable array's mechanical structure; fabrication of a full scale prototype for testing has begun. Most mechanisms have undergone significant design changes from the original baseline configuration. Figure 2 shows the most recent design of XSAS. Ten panels deploy from a $1 \mathrm{U}$ stowed volume to an overall length of $1.1 \mathrm{~m}$. The panels make a $10^{\circ}$ angle with the $\mathrm{z}$-axis once deployed to provide some structural rigidity to the array. Dynamic modeling of the deployable array will be performed using ADAMS, and will help validate new mechanism designs and aid in the characterization of the full scale extending array. The following sections detail the major mechanism design changes, preliminary testing results, and the solar panel design considerations.

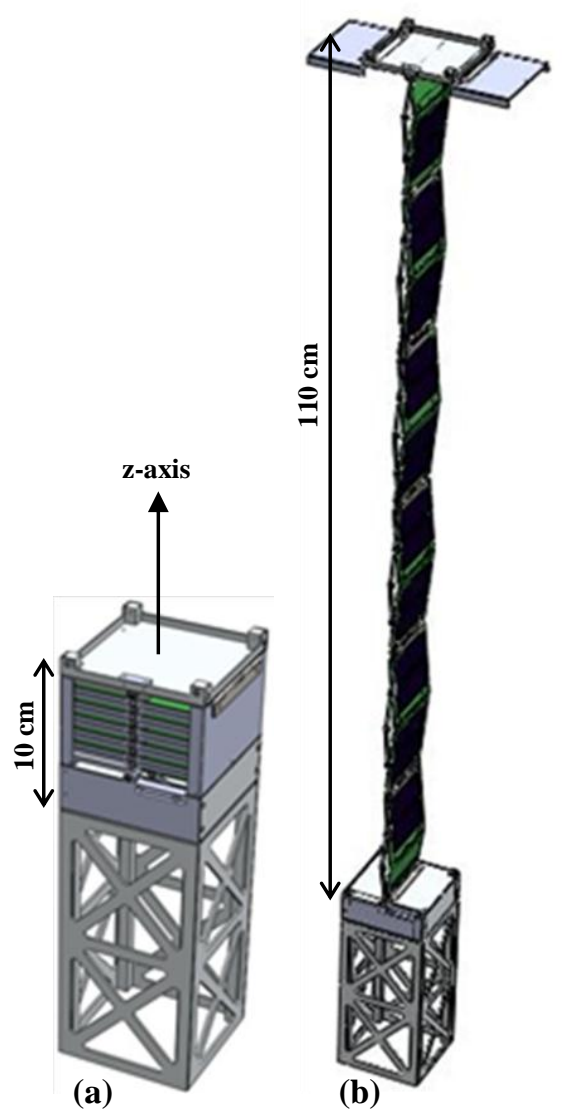

Figure 2. Third iteration of XSAS design. XSAS shown attached to $2 U$ CubeSat in a) $1 U$ stowed configuration and b) fully deployed configuration. 


\section{Mechanism Design Changes}

The XSAS baseline design has undergone some significant modifications based on lessons learned from building and testing our prototype in a microgravity environment. A new design was devised for the lower part of the scissor assembly where the latching mechanism is located. The reason for this was two-fold: stress/deformation management and mechanism volume minimization. The old latching mechanism, while experimentally verified to engage upon full XSAS extension and thus prevent the extended structure from re-compressing, was also shown to be inadequate in its lack of precision and robustness. Its flaw stemmed from a lack of minimization of the mechanism's degrees of freedom. Namely, once the bolt attached to the bottom of the scissor structure traverses the vertical slot and is brought to a stop at the end of the slot, there is a net moment on the bolt from the slot wall that causes the far side of the bolt to rotate down beyond the desired horizontal position. While it does prevent the scissor structure from re-compressing once engaged, there is still freedom for a small amount of bounce back to occur. This bounce back was confirmed through visual experimental evidence during microgravity testing ${ }^{8}$. In addition, the nature of this previous design, with its vertical displacement, constitutes a sub-optimal utilization of lower assembly volume. Based on these considerations, a horizontal constraint was devised instead of the vertical constraint. This new design allows more space for the EPS and/or additional solar panels, and also provides better constraint for the sliding shoulder bolt. Figure 3 shows a comparison of the vertical and horizontal constraint mechanism designs.
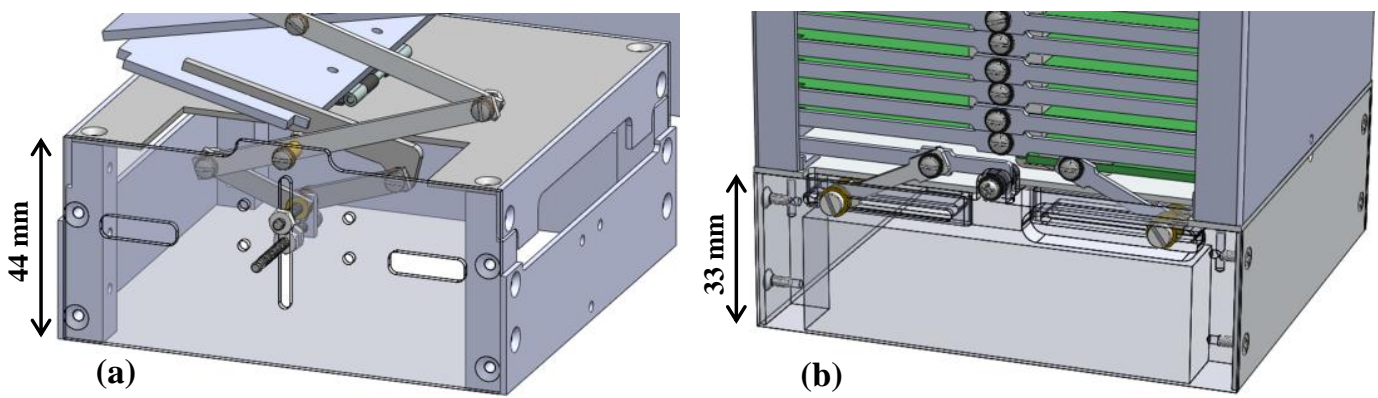

Figure 3. Comparison of vertical and horizontal constraint mechanism designs. The complicated baseline design's vertical constraint (a) requires the base to be over $1 \mathrm{~cm}$ longer and takes up more internal space than the simpler horizontal constraint design (b).

A test rig was fabricated to perform preliminary testing of the new horizontal lower scissor assembly constraint design. It consists of the lower assembly and the first two panels of the scissor structure. Using a high speed video camera, we captured the structure's deployment in detail in order to discern any high speed deformation or bounce back. A frame-by-frame view of the extension is given in Fig. 4. The test was a success in that it validated the ability of the new design to eliminate the problems documented above. There were no torques or deformations in the mechanism that restricted deployment; however, it was determined that an undesirable geometric interference occurs between two of the lower scissor components upon full extension (shown in Fig. 4 e). We have modified the final design accordingly to avoid any component interference.

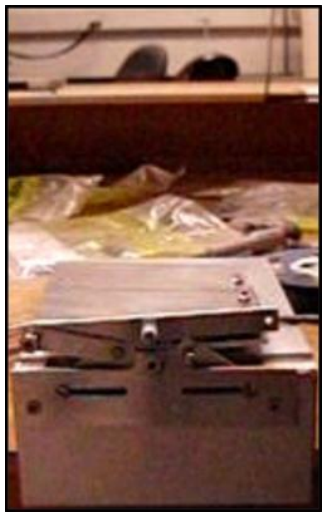

(a)

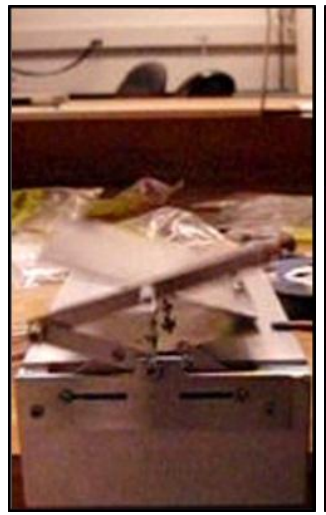

(b)

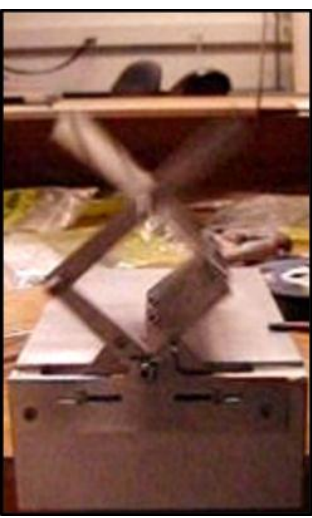

(c)

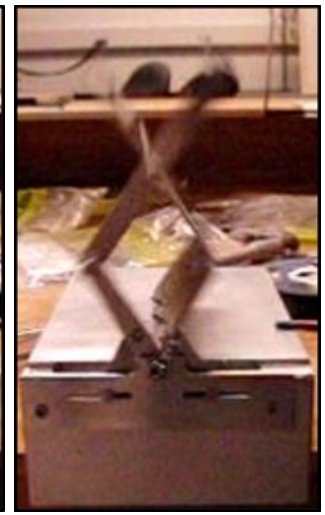

(d)

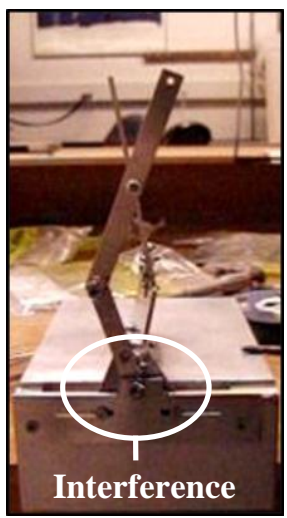

(e)

Figure 4. High speed footage of extension. Horizontal constraint design causes linear extension of panels. 
Another modification of the previous design is the panel/scissor interface. The baseline design relied upon the strength of epoxy to secure a nut in a gap at the center of each panel's edge; the scissor component was secured to this nut with a bolt. This method was found to be inadequate as the epoxy used did not provide enough shear strength to secure the nut to the panel upon experiencing the stress of multiple deployments, let alone stresses that would be experience during a launch. The new design employs a mechanical solution. A small T-shaped block is attached to the panel by two small screws, shown in Fig. 5. This threaded metal block physically secures the bolt to the panel, avoiding the use of epoxy. The T-shaped blocks also act as spacers between panels in the stowed configuration to provide support during launch. This component is currently

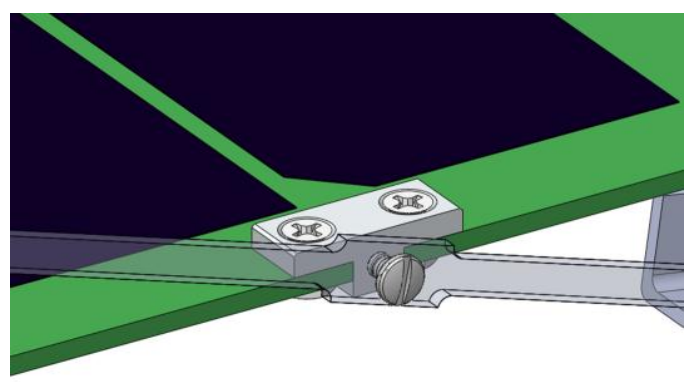

Figure 5. Improved panel/scissor interface design. Bolt from scissor arm now screws directly into T-shaped block that is mechanically attached to the panel.

The final major design change is the selection of a different spring hinge. The hinges used in past prototypes had very poor tolerances, causing unwanted twisting in the XSAS structure. A trade study was performed and a more robust spring hinge was selected. These new hinges feature a longer, higher tolerance hinge barrel that is expected to greatly reduce, if not eliminate, the twisting in the structure. These new spring hinges will be tested on the full scale XSAS structure.

\section{Solar Panel Design}

The solar panel array of XSAS will consist of a variable number of interchangeable printed circuit boards (PCBs), each with components mounted to their surface. Each board will have locations on each side for mounting solar cells, sensors, and diodes. The primary concerns for each panel are material selection and wiring management.

The two main tradeoffs for the material choice of our panels were strength and simplicity. Strength requirements are driven by a desire to reduce each panel's thickness, allowing more panels to fit inside the stowed $1 \mathrm{U}$ volume, while keeping the panels ridged enough to prevent the solar cells from cracking during launch. Simplicity requirements are driven by a desire to reduce the complexity of the entire system, thus reducing the chance of failure. Double layer PCB was chosen as the optimal medium based on deflection data from microgravity flight testing. Data taken by strain gauges on the experiments showed that maximum deflections of $1.6 \mathrm{~mm}$ thick double layer PCB were on the order of $10 \times 10^{-10} \mathrm{~mm}$ during deployment.

Use of PCB panels also allows much of the wiring of the solar array's electrical system to be integrated into the structure via wire traces. Wires carrying power and data are required to run the length of the array. Flat flex wire will connect corresponding wire traces of adjacent panels. Solar cell string length and the number of sensors used to measure panel temperature drive the number of wires between each panel. As many as 25 wires are required to interface between a single pair of panels in the 10 panel array we are designing. The panels will be designed to use solar cells with built in monolithic diodes to prevent reverse bias breakdown in the cells. Current designs are based on the Emcore BTJM III-Junction cells. To prevent a large amount of solar cell efficiency degradation, cover glass over the cell is needed. We found that the best shield thicknesses for CubeSat applications range from 30-80 microns. 


\section{B. Electrical Power System}

The EPS subsystem of XSAS focuses primarily on the interface between the solar panels and the power bus of the CubeSat it is supporting. Many aspects of this design utilize heritage from the University of Michigan's Radio Aurora eXplorer (RAX) satellite, which has many of the same functional requirements as XSAS. A high-level block diagram is given in Fig. 6 below. The following sections present an initial design of the XSAS EPS system.

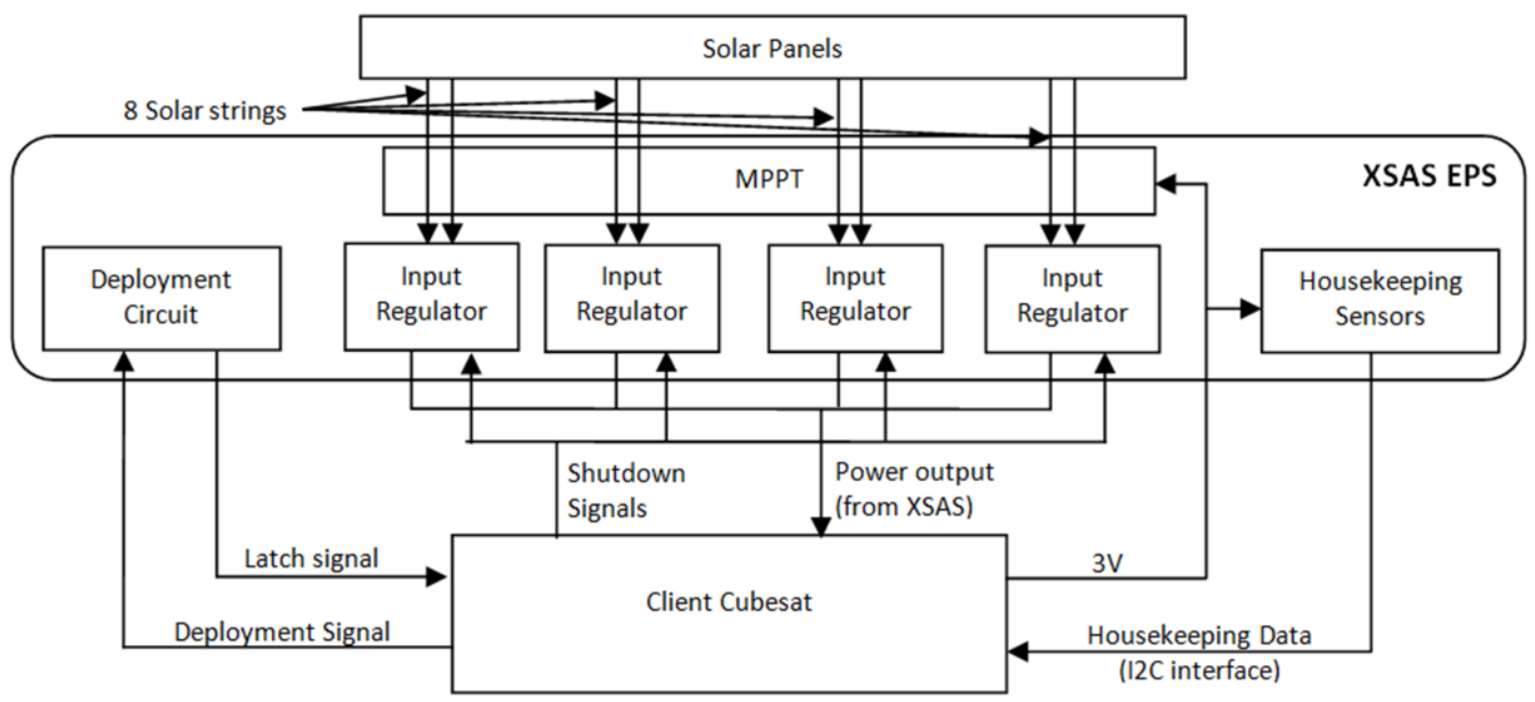

Figure 6. High-level block diagram of XSAS electrical power system.

\section{Solar Cell Strings}

The solar cells on XSAS are wired together in series into several strands known as solar cell strings. The number of strings depends on the number of solar panels present. For a full configuration of 10 panels, with 2 solar cells on each side, there will be a total of 8 strings. This number was chosen as the best trade between reliability, power generation, and wiring complexity between panels. We will consider this example as our baseline throughout this section. Figure 7 gives an example distribution of the 8 string on the lower 4 panels.

The strings are separated by the panel's angular orientation, the side of the panel the cells are mounted too, and the physical distance from the base. The choice to separate by orientation and side ensures that each string of solar panels will provide a consistent output, while the separation by physical distance reduces the need for excessive wiring across the panels. While there are a total of eight strings across the panels, only four of them will be fully active at any given time, since only one side is capable of facing the sun at any given time, allowing us to attach a pair of strings on opposing panel faces to a single input regulator.

\section{Input Regulation}

A guiding philosophy for the design of XSAS was to "build a high power generation attachment to a CubeSat, not the entire power system." If XSAS were to incorporate full EPS capabilities, along with batteries, it would be nearly $2 \mathrm{U}$ in size, leaving only $1 \mathrm{U}$ of space to the CubeSat mission it is supporting. With that in mind, only a nominal amount of regulation is performed on the power coming from the solar panels, and no secondary batteries are included on XSAS. These restrictions decrease the required circuitry, allowing more room for solar panels. Additionally, it gives mission designers using XSAS more space and flexibility when designing their CubeSat's electrical power system.

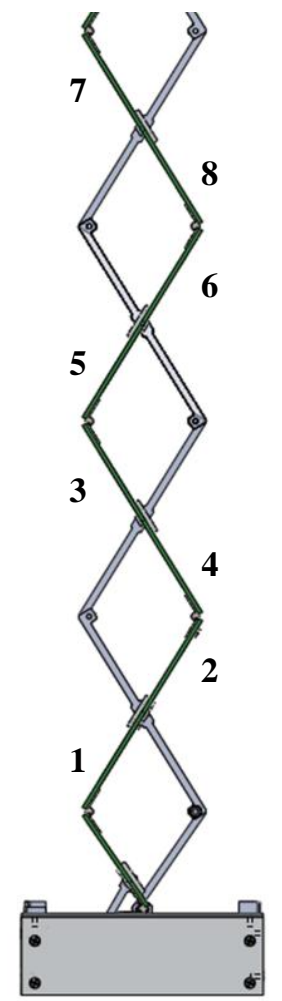

Figure 7. Example placement of the 8 strings. Strings differ by angular orientation, panel face, and distance from base. 
Power regulation is useful in order to provide a consistent power line to the CubeSat. Four input regulators, one for each string pair, are used in conjunction with a Maximum Power Point Tracker (MPPT) to provide a steady DC output to the CubeSat's power system during times of power generation. Fluctuation or disappearance of this output during times of eclipse will not be a problem as we expect XSAS will most commonly be used to charge a secondary battery array.

\section{Maximum Power Point Tracker}

In addition to input regulation, XSAS will include a MPPT to maximize the amount of power transferred from the solar panels through the input regulators. Using an Atmel AVR microcontroller, a "perturb and observe" strategy is implemented to constantly adjust the rate at which power is sent to the input regulators. As a backup, an analog set-point tracker will be included if there is any type of failure on the MPPT. The design of the MPPT will incorporate heritage from the RAX MPPT, which has many of the same functional requirements.

\section{Interface with CubeSat}

The interface between XSAS and the CubeSat is designed to provide mission designers with power and as much useful data as possible while requiring only what XSAS needs to operate. XSAS will provide a steady DC power bus. Additionally, XSAS will collect housekeeping data monitoring the temperature of the panels and the voltage and current of each string. This data will be supplied to the CubeSat through an $\mathrm{I}^{2} \mathrm{C}$ (inter-integrated circuit) interface. Inputs to XSAS include a shut-off signal for each regulator as well as one signal for initial deployment. A steady 3.3 volts direct current input to XSAS will be strongly suggested to the CubeSat developers. This input will power the housekeeping sensors and MPPT circuitry, but can be removed if mission designers can settle for only set-point tracking and no housekeeping data.

\section{Conclusion}

Valuable data gained from multiple iterations of prototyping, building, and testing XSAS in the lab and in microgravity is fundamental to the current developmental stage of XSAS technology. The technology is on its third design iteration and is focused on the development of its electrical power system, optimization of the extending solar array mechanism and intensive computer modeling and simulations. A great deal of time has been spent designing the system, manufacturing prototypes, and performing tests. It cannot be stressed enough the importance of having multiple design iterations and repeated testing and validation through computer modeling. These extensive efforts are necessary in lowering the risks inherent in designing space-based deployable systems for CubeSats and other small satellites.

Future work includes extensive prototyping and testing of the XSAS EPS, extending solar array mechanism, and structure. A full scale prototype of the XSAS system will be manufactured and used for future testing. Modeling and simulation results will be compared with additional tests performed in the lab and in microgravity environments.

The work the XSAS team does is of major benefit to the Michigan Nanosat Pipeline, and the CubeSat community in general. The ultimate goal of XSAS the project is to enable new technologies that stem from ambitious CubeSat missions requiring high power generation. Improving CubeSat technologies in this way will open the CubeSat frontier giving more universities and small companies access to space. 


\section{Acknowledgments}

The authors would like to thank the following people who have supported the XSAS program: Dr. James Cutler for providing extensive technical support and project guidance, Dr. Darren McKague for his expert advice in space systems engineering, Dr. Brian Gilchrist and the Student Space System Fabrication Laboratory for providing a place to work and years of CubeSat design experience to share, the Michigan Exploration Laboratory for their assistance in computer modeling and simulations, the University of Michigan Engineering Council for contributing to the success of our project, the Wilson Student Team Project Center and its staff for greatly helping us in the design and manufacturing process, Dan Meinzer and Scott Tripp assistance in designing our EPS and finally, we would like to thank all of the students and professors who have worked on XSAS and brought it one step closer to being a flightready vehicle.

\section{References}

1"CubeSat Design Specification Rev. 12" The CubeSat Program, Cal Poly SLO, URL: http://cubesat.atl.calpoly. edu/images/developers/cds_rev12.pdf [cited 6 March 2011].

${ }^{2}$ Long M., "A CubeSat Derived Design for a Unique Academic Research Mission in Earthquake Signature Detection," 16th Annual/USU Conference on Small Satellites, SSC02-IX-6, Logan, Utah, 2002.

${ }^{3}$ Bouwmeester J., "Preliminary mission results and project evaluation of Delfi-C3 Nano-Satellite",

${ }^{4}$ Passaretti, M., Hayes, R., 2010, "Development of a Solar Array Drive Assembly for CubeSat," Proceedings of the 40 ${ }^{\text {th }}$ Aerospace Mechanisms Symposium, NASA Kennedy Space Center, May 12-14, 2010.

${ }^{5}$ Reif, A., Hoang, V., Kalaman, A., "Recent Advances in the Construction of Solar Arrays for CubeSats," CubeSat Summer Developer's Workshop, Cal Poly, San Luis Obispo, CA, 2010.

${ }^{6}$ Silver M., Dobson B., Warren P., "Development of a Deployable Gravity Gradient Boom CubeSat", 2009 CubSat Developers Workshop, Cal Poly, San Luis Obispo, CA, 2009.

${ }^{7}$ Tethers Unlimited. "Sensor Pod". N.p., 2009. http://www.tethers.com/SensorPod.html. [Cited 5 March 2011].

${ }^{8}$ Trabert, eat al., "The eXtendable Solar Array System: A Modular Nanosatellite Power System," AIAA-2010-7654, 2010. 